\title{
Aspiration Cytopathology of Metastatic Mucinous Papillary Thyroid Carcinoma
}

\author{
Carl Morrison, M.D., Paul Wakely, Jr., M.D. \\ Department of Pathology, The Ohio State University, College of Medicine, Columbus, Ohio
}

The light microscopic histopathology and cytopathology of papillary thyroid carcinoma (PTC) and its variants is amply depicted in reviews, books, and book chapters. One of the changes in PTC, however, that is infrequently discussed or illustrated in the literature is that of mucinous metaplasia. In the aspiration cytopathology literature, we are aware of only a rare report of PTC exhibiting extensive mucinous metaplasia. We present an example correlating the histopathology and fine needle aspiration cytopathology of a PTC that had metastasized on several separate occasions to cervical lymph nodes, and in the process demonstrated mucinous transformation. Without a prior history of PTC, the aspirate smears and tissue sections could have been mistaken easily for metastatic clear cell carcinoma of non-thyroidal origin. Mucinous metaplasia represents an extremely uncommon, but nonetheless potential, pitfall in the aspiration cytopathology and histopathology of metastatic PTC.

KEY WORDS: Thyroid, Papillary carcinoma, Fine needle aspiration biopsy, Cytopathology, Mucin. Mod Pathol 2001;14(4):361-365

The presence of mucin in endocrine neoplasms is an unexpected finding in contrast to its frequent production in non-endocrine epithelial tumors. This can assume diagnostic importance if the pathologist is asked to determine the site of a mucin-producing metastatic carcinoma in a patient with either an unknown primary or a remote history of cancer. Almost four decades ago, in a large study of the surgical pathology of various metastatic neoplasms (including 93 examples of thyroid cancer), Foster et al. concluded that "the

Copyright () 2001 by The United States and Canadian Academy of Pathology, Inc.

VOL. 14, NO. 4, P. 361, 2001 Printed in the U.S.A.

Date of acceptance: October 24, 2000.

Address reprint requests to: Paul E. Wakely, Jr., M.D., Department of Pathology, The Ohio State University College of Medicine, 414 Doan Hall, 410 West 10th Ave., Columbus, OH 43210; e-mail: wakely-2@medctr.osu.edu; fax: 614-293-7626. thyroid is the only site studied that can be ruled out with great assurance when one is dealing with a mucicarmine positive metastasis" (1). Although reports to the contrary have been published (2$4)$, our impression is that many pathologists remain under the misconception that carcinomas of the thyroid are nearly all mucin-negative. This assumption from histopathology has been transferred to fine needle aspiration (FNA) cytopathology of thyroid carcinoma, where only rare descriptions or illustrations of extracellular mucin or mucin-filled vacuoles exist $(5,6)$. The aim of this article is to illustrate and describe the FNA cytopathology (and corresponding histopathology) of a mucinous papillary thyroid carcinoma (PTC) and to discuss the potential pitfalls related to this phenomenon.

\section{CASE REPORT}

A 66-year-old man had undergone complete thyroidectomy at an outside hospital for PTC 10 years earlier after an initial right cervical lymph node biopsy revealed metastatic disease. Over the past decade, he has received three courses of radioactive iodine therapy and has had two separate surgical procedures to excise repeatedly enlarged right neck lymph nodes. On each occasion these nodes contained metastatic PTC both within the node proper and in extranodal soft tissue. No metastasis beyond the right cervical lymph node group has ever been detected.

He presented to our medical center because of a third recurrence of right cervical lymphadenopathy since his initial thyroidectomy. FNA biopsy of an enlarged right cervical lymph node was performed and revealed metastatic carcinoma. Subsequent surgical resection of the aspirated node and surrounding soft tissue was performed 2 weeks after FNA confirmed that diagnosis. One year after the FNA, he presented with a fourth recurrence of a right neck mass. This was excised without performing another prior FNA, and again it revealed metastatic PTC. 


\section{Pathologic Findings}

Materials available for review included slides from two separate surgical specimens before the FNA biopsy, FNA smears, and two subsequent surgical specimens after the FNA biopsy. Aspirate smears were stained with both Papanicolaou and Diff-Quik stains. In addition to conventional hematoxylin and eosin stain, histochemical stains for mucin (mucicarmine, Alcian blue [pH 2.5], and periodic acid-Schiff stain with and without diastase digestion) were applied to tissue sections, as was immunohistochemical staining with prediluted antibodies to thyroglobulin (Dako Corp., Carpinteria, California) and calcitonin (Dako Corp.) and an in-house cocktail of three different cytokeratin antibodies (MAK-6, Zymed Corp., San Francisco, California; AE1/AE3, Boehringer Mannheim, Indianapolis, Indiana; CAM 5.2, Becton Dickinson Corp., Mountain View, California).

Aspirate smears contained many polygonal and fewer spindle cells in both flat and multilayered aggregates, and infrequently as three-dimensional branching papillae. Isolated single cells constituted a minor component of the smear. Cell nuclei were uniformly enlarged, round to oval, and had relatively smooth nuclear borders. Intranuclear inclusions (similar in color and texture to cell cytoplasm) were noticeably uncommon and difficult to locate. Papanicolaou-stained smears better highlighted the coarseness of the nuclear chromatin. Nuclei exhibited few nuclear grooves and indistinct nucleoli. Nearly all non-vacuolated cells had a moderate amount of finely granular eosinophilic (so-called metaplastic) cytoplasm, a characteristic feature of PTC (7).

The most striking and unexpected cytomorphologic feature was the presence of abundant cytoplasmic vacuolar inclusions (Fig. 1). These vacuoles were variably sized, numbered from one to several per cell, possessed a discrete sharp smooth border, and often were larger than the cell nucleus. In a minority of cells, vacuoles occupied almost the entire cytoplasmic volume. This led to indentation and displacement of the nucleus to the cell periphery, producing a signet ring appearance (Fig. 2). In addition, many vacuoles contained one or more opaque globules/concretions. These globules varied in diameter, were nearly always spherical, and showed intense metachromatic staining with the Diff-Quik stain. When present singly, they gave the cell a "targetoid" appearance (Fig. 3). Cytoplasmic vacuoles and intravacuolar globules were more transparent with the Papanicolaou stain (Fig. 4).

Tissue specimens from prior surgical specimens showed the histopathology of classic PTC metastatic to the lymph node. Careful examination of tissue slides resected 1 year before the current FNA showed only an occasional mucin-positive cell in what was otherwise typical PTC morphology. In contrast, excisional biopsy of the mass that had been aspirated 2 weeks earlier showed lymph nodes almost entirely replaced by a carcinoma with such widespread cytoplasmic vacuolization that it resembled a non-thyroidal metastatic clear cell carcinoma, or even a liposarcoma in some foci (Fig. 5). Only a few areas with non-vacuolated papillary architecture could be found, which alluded to the true nature of the metastasis. Situated within many of the cytoplasmic vacuoles were concentric eosinophilic globular inclusions identical to those described in the aspirate smears. Histochemical staining showed positive reactions for mucicarmine, periodic acid-Schiff with and without diastase, and Alcian blue in both the vacuoles and inspissated globular concretions throughout the clear cell regions (Fig. 6). Strong, diffuse cytoplasmic staining occurred with pancytokeratin antibodies in both clear cell and non-vacuolated foci. Staining with thyroglobulin was much weaker in clear cell areas and was best seen in the few non-mucinous areas of carcinoma. Immunohistochemical staining for calcitonin was negative.

Lymph nodes removed 1 year after the FNA again revealed metastatic PTC, but without the obvious clear cell areas described in the prior excisional biopsy. Although individual cells with mucinous metaplasia were present (as documented by positive reaction with the previously listed histochemical stains), these were much less obvious in this subsequent specimen and were seen only in isolated foci.

\section{DISCUSSION}

Except for medullary carcinoma $(2,5)$, most pathologists do not consider mucin production/metaplasia as part of the histopathology or cytopathology of thyroid cancers. Though PTC accounts for most thyroid malignancies, and its histo-/cytopathology are well documented, it is rarely associated with mucinous change as manifested by descriptions in major textbooks of the surgical pathology and aspiration cytopathology of PTC (8-11). Nonetheless, mucin production has been documented in both primary and metastatic PTC. Chan et al. (4) demonstrated mucin in 34/40 cases of PTC metastatic to lymph nodes. Only seven had intracellular mucin with mucicarmine-positive globules surrounded by a clear halo identical to those in our case. Mlynek et al. (3) found mucin in $45 \%$ of 142 thyroid carcinomas of various subtypes. Most exhibited only weak to moderate intensity, however. Intracellular mucin in their PTC cases had the same sharply delineated vacuoles with homogeneous eo- 


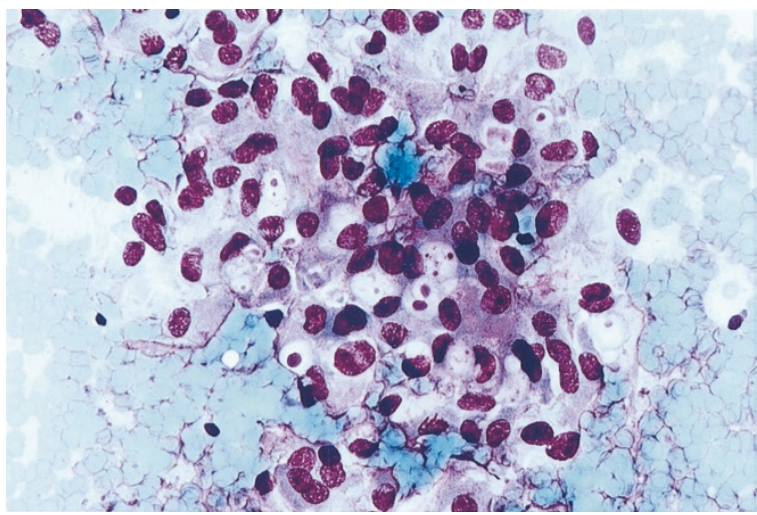

FIGURE 1. Numerous polygonal cells in this multilayered aggregate possess intracytoplasmic vacuoles, which, in turn, contain violaceous globular inclusions. Diff-Quik stain.

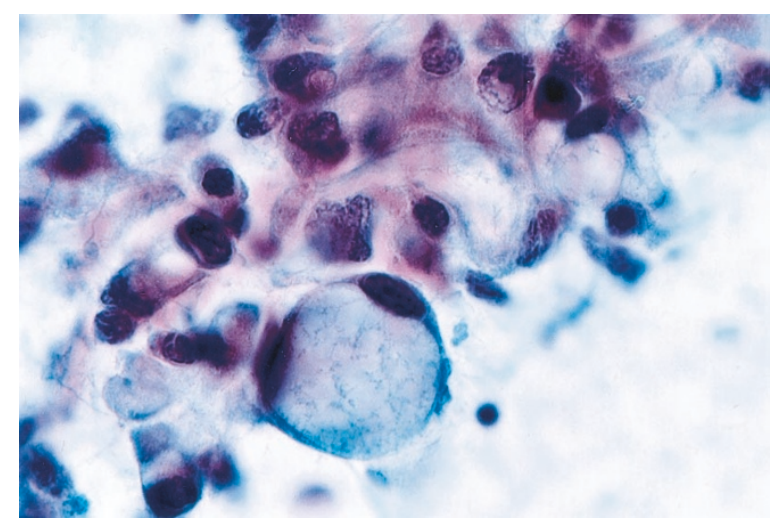

FIGURE 2. A signet ring cell is created by a large, single vacuole with the enlarged nucleus pushed to the cell periphery. Papanicolaou stain.

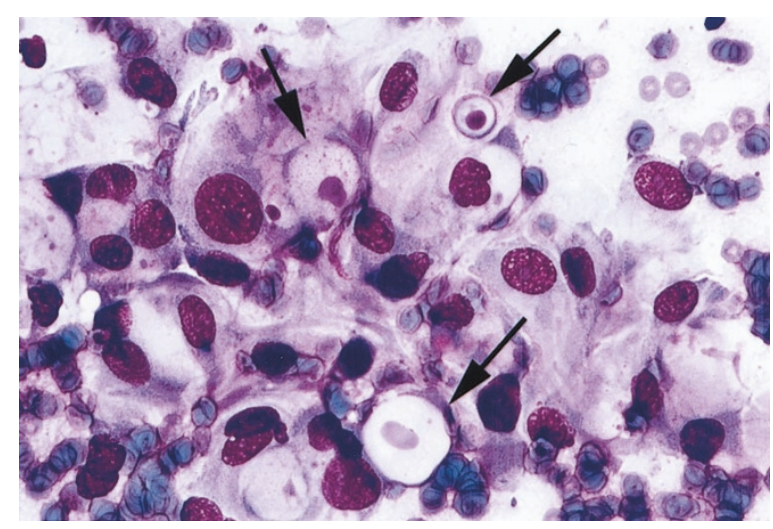

FIGURE 3. High power shows targetoid cells created by intracellular globules, each surrounded by an almost optically transparent vacuole with a sharp border (arrows). Note the cellular nucleomegaly. Diff-Quik stain.

sinophilic inclusion bodies as we have described. Alves et al. described positive immunohistochemical expression of mucin antigens (MUC1, MUC2) in a high percentage $(73 \%)$ of PTC cases, but they could not directly correlate their presence with biologic aggressiveness (12).

Differential diagnosis of mucinous metaplasia includes both lipomatous transformation and clear

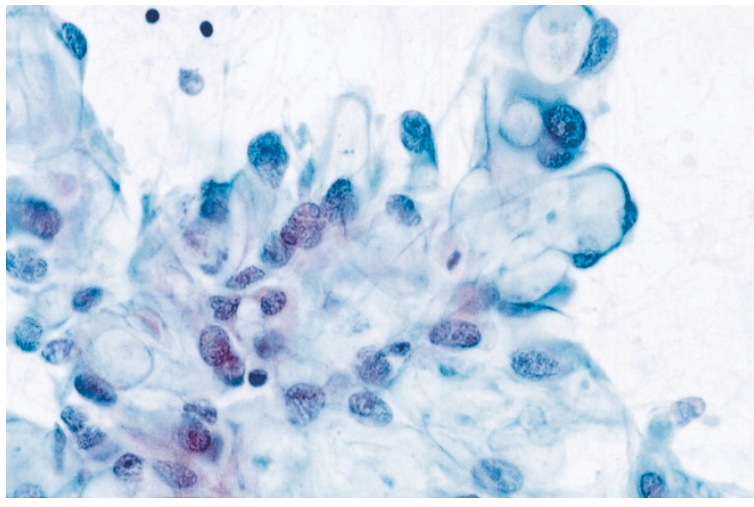

FIGURE 4. Intracellular vacuoles are easily recognized, but the globular inclusions are more transparent and, thus, occasionally imperceptible with the Papanicolaou stain.

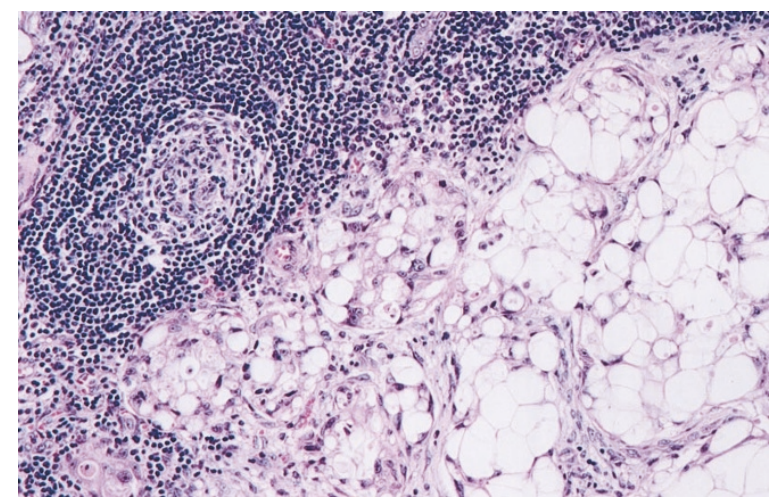

FIGURE 5. A secondary lymphoid follicle with germinal center (upper left) remains in this lymph node, which otherwise has been replaced by a coarsely vacuolated carcinoma. Eosinophilic globules are present within many of the vacuoles but are difficult to appreciate at this low magnification. Hematoxylin and eosin stain.

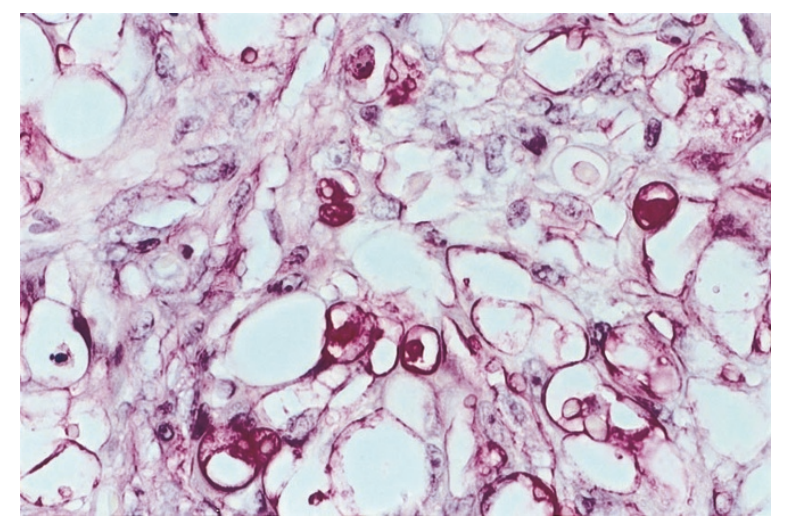

FIGURE 6. Mucin staining is positive for both the cytoplasmic vacuoles and some of the globular inclusions. Mucicarmine stain.

cell change within the thyroid. Mature adipose tissue has been described as a component of both PTC and non-neoplastic thyroid lesions in a small percentage of cases (13). In tissue sections, mature adipose tissue can be distributed as discrete aggregates within the thyroid, or it can diffusely infiltrate the gland. A diagnosis of thyrolipoma/thyroid lipomatosis should be made with caution if only iso- 
lated adipose tissue fragments are found in thyroid aspirates, because the lack of a spatial relationship with the surrounding tissue precludes determination of the exact site of these fragments. Only if follicular cells are admixed with mature lipocytes can one be more confident of this entity. In one of the rare reports of the cytopathology of thyrolipoma, such a mixture was present (14). Lipocytes had large single vacuoles with small nuclei and lacked the mucinous features described in our case.

Clear cell change also has been described in tissue sections of both benign and malignant thyroid tumors including PTC. The etiology of this clear cell change (sometimes leading to the formation of signet ring cells) has been ascribed to a variety of factors, including the accumulation of cytoplasmic vesicles, glycogen, mucin, lipid, and even thyroglobulin in some examples (15). Clear cell change rarely is described in cytopathologic descriptions of primary thyroid neoplasms, and when present one is more apt to be dealing with a metastatic tumor. Reports of metastatic renal cell carcinoma discovered by thyroid FNA biopsy as the initial manifestation of disease are not rare (16). Smears of renal cell carcinoma contain large cells in loosely cohesive aggregates and singly dispersed with abundant cytoplasm that contains multiple cytoplasmic vacuoles. Vacuoles are optically clear, variably sized but generally small (almost never attaining the same size as the nucleus), and lack the targetoid appearance seen in our example. The same form of cytoplasmic vacuolization has been described for the rare example of clear cell change in a primary thyroid neoplasm (17). So-called marginal vacuoles are small cytoplasmic vacuoles not readily confused with mucinous metaplasia. These are small, pale, bubble-like vacuoles that are peripherally situated (budding from the cell membrane), that often have a dense border, and are reliably seen only in Romanovsky-stained (Giemsa, Diff-Quik) smears. They may represent small colloid droplets, are commonly encountered in smears of Graves' disease where the term "fire flares" also has been used (11), and also have been reported in smears of follicular neoplasms (18).

Intracytoplasmic optically clear vacuoles are not uncommon morphologic constituents in diagnostic aspiration cytopathology. They have been described in aspirate smears from a broad range of neoplasms encompassing all major tumor categories and often are one of the helpful morphologic ingredients used in formulating a diagnosis. Cytoplasmic vacuoles may range from multiple fine structures within the cell (e.g., renal cell carcinoma, Burkitt lymphoma, Ewing sarcoma), to single signet ring-type vacuoles (e.g., gastric carcinoma, mesothelioma), to large, peripherally placed blisterlike projections that distort the outline of the cyto- plasmic border (e.g., seminoma). A peculiar type of cytoplasmic vacuole that harbors a central circular concretion often has been referred to as a "target" or "targetoid cell." Although such a cell has long been recognized as characteristic of breast carcinoma (particularly lobular carcinoma) (19), and is encountered in tissue sections and aspiration smears alike, it is not unique to that malignancy. Nonetheless, because targetoid cells are commonly associated with breast carcinoma, their presence in metastatic deposits has been found to be diagnostically profitable by several authors in directing attention to the breast as the site of origin (20-22). The spherical intravacuolar globule is thought to represent inspissated granular mucin, and ultrastructurally it is an electron-dense body surrounded by a vacuole, which, in turn, is lined by uniform microvilli (20).

Similar to tissue pathology, the FNA biopsy literature has nearly always associated "targetoid" cytoplasmic vacuoles with breast carcinoma with few exceptions. To our knowledge, only rare examples of mucin-laden vacuoles in aspirate smears of thyroid carcinoma have been reported. Haleem et al. describe a case of medullary thyroid carcinoma with intracytoplasmic vacuoles containing amorphous mucin (5). Discrete intravacuolar targetoid globules are not illustrated in their example, although electron microscopy demonstrated occasional vacuoles lined by microvilli. As part of a discussion of diagnostic dilemmas in thyroid cytology, Galera-Davidson illustrates an example of PTC with mucinous intracytoplasmic vacuoles but without the targetoid-type inspissated concretions (6). Some authors in their discussion of breast carcinoma aspirates have referred to this targetoid structure as a "magenta body" because of its deep violaceous coloration and its distinctive appearance in air-dried Diff-Quik-stained smears (23). Targetoid cellular vacuoles (magenta bodies) were present throughout the smears in our example of metastatic mucinous PTC. Had one not been aware of the history, it is doubtful that a metastasis from the thyroid would have been contemplated from the cytomorphology alone. Metastatic carcinoma from the breast, lung, salivary gland, or stomach would have been considered much more likely candidates as a primary source.

Our report emphasizes that metastatic PTC has the rare capacity for intracellular mucin production, which can appear as targetoid intracellular mucin vacuoles morphologically identical to those encountered more commonly in FNA smears of breast carcinoma. Careful clinical history and corroborating immunohistochemistry may be necessary to completely eliminate metastatic PTC from consideration when such structures are seen in aspirates of the head and neck. 


\section{REFERENCES}

1. Foster EA, Levine AJ. Mucin production in metastatic carcinomas. Cancer 1963;16:506-509.

2. Zaatari GS, Saigo PE, Huvos AG. Mucin production in medullary carcinoma of the thyroid. Arch Pathol Lab Med 1983; 107:70-74.

3. Mlynek ML, Richter HJ, Leder LD. Mucins in carcinomas of the thyroid. Cancer 1985;56:2647-2650.

4. Chan JKC, Tse CC. Mucin production in metastatic papillary carcinoma of the thyroid. Hum Pathol 1988;19:195-200.

5. Haleem A, Akhtar M, Ali MA, Iqbal Z. Fine-needle aspiration biopsy of mucus-producing medullary carcinoma of thyroid: report of a case with cytologic, histologic and ultrastructural correlations. Diagn Cytopathol 1990;6:112-117.

6. Galera-Davidson H. Diagnostic problems in thyroid FNAs. Diagn Cytopathol 1997;17:422-428.

7. Miller TR, Bottles K, Holly EA, Friend NF, Abele JS. A stepwise logistic regression analysis of papillary carcinoma of the thyroid. Acta Cytol 1986;30:285-293.

8. Merino MJ, Sidawy MK. The thyroid gland. In: Silverberg SG, DeLellis RA, Frable WJ, editors. Principles and practice of surgical pathology and cytopathology. 3rd ed. New York: Churchill Livingstone; 1997. p. 2665-708.

9. LiVolsi VA, Montone K, Sack M. Pathology of thyroid disease. In: Sternberg SS, Antonioli DA, Carter D, Mills SE, Oberman HA, editors. Diagnostic surgical pathology. 3rd ed. Philadelphia: Lippincott Williams \& Wilkins; 1999. p. 529-87.

10. Kini SR. Guides to clinical aspiration biopsy. Thyroid. 2nd ed. New York: Igaku-Shoin; 1996.

11. Orell SR, Sterrett GF, Walters MN, Whitaker D. Manual and atlas of fine needle aspiration cytology. 3rd ed. London: Churchill Livingstone; 1999.
12. Alves P, Soares P, Rossi S, Fonesca E, Sobrinho-Simoes M. Clinicopathologic and prognostic significance of the expression of mucins, simple mucin antigens and histo-blood group antigens in papillary thyroid carcinoma. Endocrine Pathol 1999;4:305-313.

13. Gnepp DR, Ogorzalek JM, Heffess CS. Fat-containing lesions of the thyroid gland. Am J Surg Pathol 1989;13:605-612.

14. Rollins SD, Flinner RL. Thyrolipoma: diagnostic pitfalls in the cytologic diagnosis and review of the literature. Diagn Cytopathol 1991;7:150-154.

15. Carcangiu ML, Sibley RK, Rosai J. Clear cell change in primary thyroid tumors. A study of 38 cases. Am J Surg Pathol 1985;9:705-722.

16. Halbauer M, Kardum-Skelin I, Vranesic D, Crepinko I. Aspiration cytology of renal cell carcinoma metastatic to the thyroid. Acta Cytol 1991;35:443-446

17. Sauer T, Olsholt R. Clear cell follicular adenoma of the thyroid: a case report. Diagn Cytopathol 1996;15:124-126.

18. Volavsek $M$, Us-Krasovec $M$, Auersperg $M$, Hocevar $M$, Golouh R. Marginal vacuoles in fine-needle aspirates of follicular thyroid carcinoma. Diagn Cytopathol 1996;15:93-97.

19. Foote FW Jr, Stewart FW. A histologic classification of carcinoma of the breast. Surgery 1946;19:74-99.

20. Battifora H. Intracytoplasmic lumina in breast carcinoma. A helpful histopathologic feature. Arch Pathol 1975;99:614-617.

21. Andersen JA, Vendelboe ML. Cytoplasmic mucous globules in lobular carcinoma in situ: diagnosis and prognosis. Am J Surg Pathol 1981;5:251-255.

22. Quincey C, Raitt N, Bell J, Ellis IO. Intracytoplasmic lumina-a useful diagnostic feature of adenocarcinomas. Histopathol 1991;19:83-87.

23. Linsk JA, Franzen S. Breast aspiration. In: Clinical aspiration cytology. Philadelphia: J.B. Lippincott Co.; 1983. 